\title{
La Valoración de la Salud: elemento esencial del desarrollo
}

\author{
Ezequiel Jaimes Figueroa ${ }^{1}$ \\ Coordinación General de Investigación y Estudios Avanzados, \\ UAEM
}

\section{Introducción}

El desarrollo, entendido como un fenómeno social complejo, que se desenvuelve dentro de dimensiones sociales, políticas y culturales, delimitadas por el ámbito de su propio crecimiento y posibilidades, y regulado por el progreso científico; establece relaciones entre sus diversos componentes como la salud en dos sentidos: el impacto que produce el desarrollo sobre el estado de salud, y el impacto que ejerce la salud sobre el propio desarrollo.

El desarrollo (particularmente el desarrollo económico) ha contribuido a mejorar la salud y extender la vida, aún antes de la existencia de la medicina moderna, a tal grado que han propiciado la aparición de la llamada "transición epidemiológica" que implica que una sociedad más desarrollada se caracterice por una baja incidencia de muchas enfermedades infecto-contagiosas, y una mayor incidencia prevalencia y mortalidad de enfermedades cardiovasculares: neoplásicas y crónico-degenerativas.

Los principales mecanismos responsables de esa reducción en la mortalidad probablemente hayan sido el mejoramiento de la dieta, una mayor estabilidad en la oferta alimentaria (asociada a los avances en el transporte), la disminución de la fertilidad, y en general, el ingreso. Este último, es el factor más estudiado y del que hay que distinguir dos elementos fundamentales:

a) la diferencia entre el ingreso individual y colectivo, $y$

b) la forma de la relación entre el nivel de ingreso y el estado de salud.

Esta percepción del ingreso ha conducido a entender que existe un nivel de pobreza absoluta debajo del cual es imposible mantenerse vivo, yen el otro extremo, hay una expectativa de vida máxima o de saturación que es independiente del nivel de ingreso (relación ingreso/esperanza de vida). Sin embargo, hay que destacar que en determinados grupos poblacionales, en ciertas

$1 \mathrm{El}$ autor es Coordinador General de Investigación y Estudios Avanzados de la UAEM. 
épocas y circunstancias, pueden ser tanto o más importantes los desarrollos culturales, en el sentido de cambio de conocimientos y de hábitos.

El desarrollo contribuye a mejorar la salud, pero no todos los efectos del desarrollo sobre la salud son beneficiosos para esta, por lo que el estilo de desarrollo destaca tanto en la composición de la producción como de su distribución geográfica, lo que implica la compensación de los efectos del desarrollo insalubre.

El análisis histórico de la mortalidad ha propiciado la conclusión de que la salud depende esencialmente de las condiciones de vida; además de que los servicios de salud no han jugado un papel más allá de lo marginal. Sin embargo, a partir del principio de este siglo, los sistemas de salud han desempeñado una función importante debido a que la relación ingreso/estado de salud solo es determinante a bajos niveles socioeconómicos. Desde esta perspectiva, se ha pro- puesto que la medicina moderna genera enfermedad e incapacidad, desvía las acciones preventiva a un conjunto de acciones curativas, en tanto que la propia realidad ha manifestado que la medicina moderna es un factor contribuyente a la salud, reflejándose necesariamente en el estilo de desarrollo, orientándose al análisis del tipo de desarrollo y del tipo de atención de salud.

\section{La salud como capital humano}

La relación entre el desarrollo y la salud parece clara, pero también existe el sentido inverso, es decir, el impacto que produce la salud sobre el desarrollo, por lo que este enfoque orienta al análisis del "capital humano", del cual la salud forma parte. En este sentido, cada ser humano representa un "capital" como la suma de sus capacidades, de todo lo que sabe y puede hacer, sobre el que la enfermedad, la incapacidad o la muerte reducen este potencial. Siendo así, cualquier gasto para defender o restaurar la salud puede ser una inversión. Generalmente, el concepto de "capital humano" se refiere a la capacidad de producir económicamente, la cual se mide o se valoriza por el ingreso adicional generado.

Desde esta perspectiva, no es extraño que la buena salud contribuya al desarrollo y que la mala salud sea un impedimento a ello; lo extraño es que la evidencia empírica sobre este punto sea todavía limitada y algo contradictoria, pues desde la perspectiva de la relación salud-desarrollo, no se ha encontrado una contribución consistente que haya realizado la salud al desarrollo. Esto se explica debido a que el costo de la enfermedad no aparece en la forma de ingreso perdido, sino en la pérdida de consumo, de ocio, o en la forma de 
más trabajo para llegar al mismo producto; lo que ha propiciado la sugerencia de que ciertas acciones para mejorar la salud tengan efectos negativos sobre el desarrollo como es el caso del incremento en el número de dependientes a expensas de los ancianos.

En tanto que el estado de salud es una forma de capital, implícitamente comparte con cualquier capital físico el estar expuesto a riesgos imprevistos y a deteriorarse con el tiempo; además es un capital natural, pues nace con todo individuo; es un capital inalienable, debido a que puede comprar un capital físico nuevo pero no un cuerpo nuevo; finalmente, es un capital que contribuye a definirnos como seres humanos.

Cualquier concepto de igualdad intrínseca de los seres humanos está en contradicción con aceptar que el capital humano en forma de salud también participe en la pobreza económica. De esta preocupación, se deriva la costumbre de subsidiar los servicios de salud, no solo por parte de los sanos en favor de los enfermos, sino también subsidios de los ricos en beneficio de los pobres. El reconocimiento de este hecho y la socialización de recursos para defender la salud más allá de lo justificado por su rendimiento económico, son elementos del desarrollo de una sociedad, expresados a través de su sistema político.

Este enfoque conduce necesariamente a la valoración de la vida, predominando el enfoque en el que es deseable asignar un valor monetario a la vida humana en el sentido de fijar una cantidad específica y definida como el gasto requerido para prevenir una muerte prematura. A partir de esta idea, el Gobierno puede tomar decisiones relativas a cuánto, en qué, cuándo y donde invertir en servicios de salud, sin embargo, es necesario tener presente que desde esta perspectiva, se asigna un mayor valor a la vida de algunas gentes que de otras.

Con el propósito de analizar el valor de la vida y así tener variables para la toma de decisiones, se han construido algunos indicadores que se basan en la suma de capital equivalente a los ingresos netos de las personas a las que se puede salvar su vida; y muchos economistas favorecen los indicadores calculados a partir de las cantidades que la gente puede gastar para evitar un incremento en la probabilidad de la muerte; sin embargo, estudios empíricos han demostrado que el "valor de la vida" puede incrementarse con el ingreso, por lo que la valoración de la vida humana en términos monetarios sugiere necesariamente plantearse la siguiente interrogante: ¿cuánto estamos preparados para gastar?

La evaluación económica del valor de la vida, está asociado al análisis costo-beneficio, en el que la vía comúnmente empleada es un valor moneta- 
rio; pero es importante referir que la vida humana valorada en el verdadero sentido de la palabra no puede tener un precio en sí misma.

Determinar cuánto cuesta salvar la vida humana es un problema que día a día va cobrando mayor vigencia, problemática que es valorada a partir de muchos elementos; entre los que se encuentran la evaluación económica de la vida como un ingreso dentro del estándar de vida de la población, o bien, los gastos para la prevención de enfermedades susceptibles de evitarse y sobre todo, donde no se está preparado para gastar cantidades infinitas de dinero.

Los estudios de Gavin Mooney han concluido que las cantidades gastadas por los gobiernos para salvar una vida varían grandemente entre diversos países, lo que puede ser una diferencia genuina en la inconsistencia de planes de gastos del gobierno o un hecho genuino de la diferencia en el tipo de vida salvada. Por otra parte, la población no percibe que la pérdida de la vida involucra situaciones diferentes, en personas diferentes, en tiempos y lugares diferentes, por lo que las grandes inversiones de gastos parten del desconocimiento de la identidad de la persona involucrada para gastar dinero para salvar vidas; asunto que se debate dentro de la moralidad, lo religioso o lo ético.

Hay otra área relevante a la política donde la gente parece observar diferentes situaciones involucradas en la muerte con diferente seriedad, áreas que son explotadas por diversos grupos de presión que discuten si pueden o no tomar acciones para prevenir la muerte, lo que implica diferentes clases de muerte y en consecuencia dificulta cualquier argumento racional, atribuyéndole al gobierno una inherente inmoralidad. Desde esta perspectiva, es más defendible - al parecer - que el gobierno en su toma de decisiones pueda tener presente las diferentes actitudes hacia diversos "tipos de muerte", permitiendo que los gobernantes consideren las diferentes actitudes de la población hacia los diferentes problemas de salud o de la población involucrada en situaciones potencialmente peligrosas; por lo que la selección de donde gastar dinero para salvar vidas, es tan desagradable que hay una tentación obvia para evitarlo.

La declaración de que el dinero y la vida son inconmensurables puede tener varias implicaciones y significados y constituyéndose en una apreciación para fundamentar la toma de decisiones; aún cuando haya otras bases "raciona- les" (no monetarias) para tomar decisiones sobre cuanto gastar para salvar la vida humana; o bien, puede reclamarse que las decisiones involucradas en la salvación de la vida humana, o permitir que la población muera, es siempre tan complejo que se pueden establecer principios generales, desenvolviéndose, sin lugar a dudas, en el ámbito de la bioética. 
Cuando se acepta que para algunos propósitos deseamos asignar un valor monetario a la vida, tenemos una respuesta a la pregunta de cuánto puede ser este valor. Mientras el método más popular para asignar un valor monetario a la vida ha considerado las ganancias netas de impuestos de la gente, también se puede considerar la suma del capital que puede producir un nivel de ganancias, y trata a este capital como el valor económico de la persona. Esto sugiere que se puede analizar cuánto del resto de la comunidad puede estar preparado para gastar en salvar una vida, debido a que puede deducirse el consumo de los ingresos de la persona involucrada, sin dejar de estar representada su contribución en el conjunto de la comunidad.

El capital humano se aproxima a la valoración de la vida, por ejemplo, el capital que suma lo que puede dar la ganancia de los involucrados, parece un principio básico del análisis de costo-beneficio. Lo que deriva en la interrogante de cuanto puede ser preparado para pagar en orden a tener su vida salvada, o usando la pregunta equivalente, cuánta compensación puede ser requerida en orden para perder esas vidas. Las bases de los estudios de costobeneficio son valiosas por sí mismas en términos de que la gente puede estar preparada para pagar para reducir las muertes prematuras, o lo que pueden requerir como compensación, si se presentan situaciones peligrosas fatales.

Una aproximación de la valoración de la vida humana basada en la elaboración de decisiones, consiste en la realización de acciones que no responden a ninguna particularidad individual para aceptar ciertas muertes si un proyecto para salvar vidas o reducir riesgos es o no implementado, lo que implica, necesariamente, muchos propósitos políticos. En este orden de ideas, los cambios de contexto para juzgar el valor de una vida en particular requieren comprobar el valor de la alteración en la oportunidad de muerte, permitiendo que la valoración esté dentro de la aproximación general del costo-beneficio.

Aunque muchos economistas coinciden con la valoración en términos monetarios de la vida humana, existen algunas excepciones. John Broome explica que la vida humana es inconmensurable con la valoración monetaria, además de destacar la relación entre la posibilidad de una necesidad de cuánto gastar y la exposición a situaciones con un riesgo incrementado. Esto ha motivado plantear diversos problemas en términos de riesgo más que en términos de ciertas muertes en lo particular, propiciando que se identifique una diferencia esencial entre una acción tomada que involucra un pequeño riesgo elevado de muerte y una acción gubernamental que involucra un amplio número de gente en un pequeño riesgo de muerte de cada uno de ellos; es decir, existe una diferencia entre la elaboración de decisiones entre individuos y el gobierno. 
Existen dos argumentos que pueden indicarse contra las ideas de Broome, en relación a defender la aproximación de la valoración de la vida al observar cuanto gasta la gente para evitar riesgos. La primera es que la crítica de la valoración de la vida por estudios de costo-beneficio no ofrece ninguna alternativa. La segunda es que hay una diferencia al final entre el bajo registro de víctimas anónimas de la política y la baja valoración dada por el desconocimiento del riesgo.

Si nosotros empleamos el valor que la gente puede dar a una reducción en el riesgo de sufrir una muerte como una base para nuestra decisión, el siguiente problema es cómo extraer y medir este valor en particular. Una respuesta es la llamada "aproximación al 'cuestionamiento' del problema”, en el que el individuo responde a la interrogante sobre cuánto esta preparado para gastar de manera extra e inesperada. Otra posible vía de valoración de la vida en función del riesgo es la que involucra pagos a la gente que toma un trabajo riesgoso, pago que está en función de los diferentes tipos de nesgo.

La vinculación salud-desarrollo implica unir otros puntos además de los planteados en el análisis costo-beneficio o en otros modelos para la toma de decisiones. El primero es que el valor dado se basa en la compensación demandado por la gente que está expuesta al riesgo. El segundo es que los estudios empíricos solo incluyen riesgos de accidentes de trabajo fatales. Así, la figura mostrada, solo puede ser usada en toma de decisiones sobre proyectos donde la relación entre lesiones y muerte es probablemente similar a los accidentes en el trabajo. El análisis de ambos esquemas parece indicar que es necesario asignar alguna valoración a la salvación de la vida con el propósito de obtener decisiones adecuadas a las necesidades de la población. Esta valoración es por sí misma consistente con la aproximación que permiten los estudios de costo-beneficio, cuando se considera la compensación requerida en un riesgo incrementado de muerte, o cuando pagan para evitar tal incremento de riesgo.

\section{El gasto en atención de salud}

La relación ingreso/salud es importante a niveles bajos de ingreso y tiende hacia la independencia entre las dos variables. La relación ingreso/gasto-ensalud muestra una fuerte conexión a cualquier nivel de ingreso, es decir, el ingreso nunca deja de tener influencia en los gastos en salud, ni para un individuo ni para una sociedad. 
En este sentido, el gasto sube tanto o más rápidamente que el ingreso. Por otra parte, el proceso de decisión de cuánto gastar y en qué, es complicado, por lo que la relación admite más variaciones en el tiempo que en el espacio, convirtiendo la mejoría de la salud en un bien privado, más que social.

Así, la cuestión económica ha tenido una importancia creciente en el terreno de la atención de la salud a medida que ha aumentado la participación del sector en el producto nacional bruto. Ciertas acciones de la atención de la salud son rentables para la sociedad como un todo, en el sentido de que el valor presente del flujo de gastos en que incurre resulta menor que el valor presente del flujo de ingresos adicionales generados.

Una justificación económica falla en particular en los: ancianos, los niños menores, los minusválidos, y los pobres; por lo que las razones de gastar en atención de salud no se limita a una sola justificación: involucro las decisiones privadas en relación al ingreso individual, la socialización de riesgos a través de seguros privados o públicos, la rentabilidad de inversiones en el capital humano de una sociedad y los factores éticos que están por detrás de los subsidios redistributivos de ricos a pobres.

Actualmente, se ha incrementado, por un lado, la preocupación por contener el costo de la atención de la salud, y por otro, la promoción de medidas específicas para lograr un uso más eficaz de los recursos. Así se han integrado sistemas como el Medicare de los Estados Unidos, el auto seguro y contratación de servicios específicos, y por otro, la preocupación por el uso prudente del médico de una gran proporción del producto nacional bruto.

En respuesta a estas inquietudes, Drummond y Cols han propuesto diez nociones básicas relacionadas con la Economía de la Salud:

1. Las necesidades humanas son ilimitadas pero los recursos son finitos: las necesidades de salud no se eliminarán jamás y están continuamente redefiniéndose. Además, los recursos empleados en salud, tienen otros usos beneficiosos fuera y dentro del sector salud. Así son inevitables las decisiones respecto de la asignación de recursos escasos a actividades en competencia unas con otras.

2. La economía tiene que ver tanto con los beneficios como con los costos: dadas las limitaciones de los recursos, hay que elegir entre actividades beneficiosas que compiten con otras en los niveles de planificación y clínico: El costo real de emprender una actividad específica es el beneficio al no aplicar los mismos recursos a otro uso óptimo; por lo tanto, los economistas están interesados en los beneficios, en la eficiencia. 
3. Los costos de los programas y tratamientos de salud no están restringidos al hospital, ni siquiera al sector de salud: gran parte de los esfuerzos para aumentar la eficiencia se ha concentrado en la atención secundaria y terciaria; pero día a día se incrementan los programas a nivel de atención de primer nivel, en el que no solo participan instituciones del sector salud, sino también otros organismos públicos y privados.

4. Las decisiones en materia de atención de la salud (planificación de la salud o modalidad de tratamiento) entrañan inevitablemente juicios de valor: al analizar los problemas de atención de la salud, es importante la cuestión acerca de qué valores debe usarse al adoptar decisiones en la materia.

5. Muchas de las reglas sencillas de operación del mercado no se aplican en el campo de la atención de la salud: si los mercados funcionan bien, los precios de los recursos (y de los productos) reflejan sus costos sociales de oportunidad produciéndose eficazmente, puesto que frente a la competencia, los productores ineficientes tendrían que liquidar sus negocios (eficiencia técnica), en tanto que los recursos escasos se asignarán de tal modo que permita satisfacer las necesidades de la población (eficiencia de asignación). En el campo de la salud, se destaca:

a) Dada la incapacidad técnica de los consumidores (pacientes), los prestadores de servicios (médicos) son una pieza fundamental para determinar la demanda de atención (relación de agencia).

b) Los consumidores no pagan un precio que refleje el costo de oportunidad social del recurso usado (los consumidores experimentan un "riesgo moral").

c) El beneficio de una persona que recibe servicios, puede no estar limitado a esa persona (argumento de la externalidad).

6. La consideración de los costos no es necesariamente un recurso poco éti$c o$ : El dilema de proporcionar una atención médica teniendo presente los costos de la misma, implica realizar una distinción entre las decisiones médicas hechas en nombre de un paciente y aquellas hechas en nombre de un grupo de pacientes.

7. La mayor parte de las decisiones en cuanto a la atención de la salud se relacionan con cambios en el nivelo alcance de una actividad dada. la evaluación pertinente concierne a estos cambios marginales, no a la actividad total: en el campo de la atención de la salud a menudo hay una tendencia equivocada a presentar decisiones basadas en una 
disyuntiva de todo o nada; pues, la cuestión no es si hacemos $\mathrm{X}$ sino más bien cuánto, es decir, los costos y beneficios marginales se relacionan estrictamente con una unidad más o menos de producción, pero con frecuencia se usan para referirse a los costos y beneficios adicionales del cambio en la escala de actividad.

8. La prestación de servicios de atención de la salud es sólo una forma de mejorar la salud de la población: el estudio de esta noción sitúa en perspectiva todos los esfuerzos dentro del sistema de atención de la salud.

9. Como comunidad preferimos postergar los costos y adelantar los beneficios: diferentes inversiones en atención de la salud tienen perfiles de tiempo diferentes en cuanto a los costos y los beneficios, en tanto que otras inversiones entrañan una corriente uniforme de costos, con un rendimiento rápido desde el punto de vista de un mejor estado de salud para los pacientes tratados; es decir, existe una tasa positiva de preferencia cronológica, por lo que las inversiones en atención de la salud necesitan compararse de acuerdo con una base común si es que sus perfiles de costos y beneficios difieren.

10. La equidad en la atención de la salud puede ser deseable, pero la reducción de las inequidades generalmente tiene un precio: este argumento se centra en las relaciones de externalidad en la salud, es decir, existe una preocupación por la equidad en el suministro de atención de la salud, por exposición al riesgo, clase de ingresos, clase social, ubicación geográfica, y necesidades.

\section{Un ejemplo aplicado de la relación entre salud y desarrollo}

En busca de métodos que apoyen la toma de decisiones y maximizar el beneficio al priorizar necesidades, se han realizado diversos trabajos que pretenden correlacionar múltiples indicadores de salud que llevan implícitos los conceptos del capital humano y la valoración de la vida humana para la toma de decisiones en el gasto e inversión en salud.

Algunos de estos, como el de Bustamantes-Montes y Cols, realizados en México, se orientan a emplear un modelo de inversión-producción-consumo (IPC) que intenta cuantificar el impacto socioeconómico de la mortalidad. Este tipo de evaluación toma en cuenta la edad al morir y la potencial productividad futura generando así pérdidas o ganancias a la sociedad, dependiendo de la etapa de la vida en que ocurrió la muerte. Se ilustra para el caso de México el impacto de las diferentes causas de muerte y se compara con la importancia 
relativa a partir del ordenamiento con tasas de mortalidad y el indicador de años de vida perdidos. El ordenamiento por tasas muestra a las enfermedades del corazón, accidentes y tumores como las primeras causas. Por otro lado el indicador de años de vida potenciales perdidos tradicional muestra en primer lugar a las causas de muerte en menores. El indicador de años de vida productivos perdidos, sin embargo, concede mayor importancia a las infecciones, a los accidentes y los homicidios desde el punto de vista de producción y de consumo.

En la planeación de los servicios de salud, la mortalidad es y ha sido un indicador para medir la salud de una comunidad, por su rápido y fácil acceso, además también sirve para la asignación de los recursos a los programas de las principales causas de muerte. Sin embargo estos estudios destacan la necesidad de buscar otros indicadores, asignándoles peso y valores diferentes, tratando de prever una imagen más precisa de las causas de mortalidad.

En este trabajo se emplean los años de vida productivos perdidos según el modelo de inversión-producción (A VP- Pipc), adaptado de Gardener y Sanboin. Este modelo utiliza las defunciones según el balance entre la inversión ( 0 a 14 años de vida), producción ( 15 a 64 años de vida) y el consumo (65 y más años de vida).

Se dice que el orden de importancia de las causas de muerte, cambia según el método que se utiliza para su ordenamiento; así tenemos que:

a) Ordenamiento por tasas: equivale a asignar el mismo peso o valor a cada muerte, independientemente de la edad en que ocurre la defunción.

b) Ordenamiento por indicador de años de vida potencial perdidos tradicional: muestra en primer lugar las causas de muerte en edades menores.

c) Ordenamiento de años de vida productivos perdidos: son importantes para este indicador todas aquellas pérdidas en la edad productiva.

Los resultados obtenidos en este estudio muestran que la importancia relativa de las causas de muerte cambia al ser usado indistintamente uno $\mathrm{u}$ otro ordenamiento.

Es claro que ningún indicador es suficiente para priorizar o para asignar un valor a la vida humana, ya que la muerte es diferente para cada sociedad y para una misma, por los diferentes valores que se le puede dar durante las etapas de la vida inversión, productiva o de consumo.

\section{Conclusión}

El enfoque del capital humano para interpretar a la salud como la ausencia de enfermedad o incapacidad física o mental, junto con una expectativa de 
vida próxima al máximo biológico, esto es, la ausencia de muerte prematura por cualquier causa, implica necesariamente la reflexión ética del quehacer médico, del propio bienestar social, derivado propiamente del proceso de desarrollo del país.

Sin embargo, además del contexto ético que supone, el análisis de costobeneficio o de cualquier otro sistema para asignar prioridades a la inversión en salud, destaca que no es una medida eminentemente intrínseca a las instituciones gubernamentales, sino que deben gestionarse los mecanismos a través del cual, la propia comunidad pueda proporcionarse un cuidado y mejoramiento de su estado de salud.

En contraposición a la imagen convencional del Estado como proveedor de servicios de salud, se asiste hoya una serie de alternativas que enmarcan la salud dentro de un contexto delimitado por el bienestar social, la participación social y política de la sociedad, y la economía de mercado; por lo que entender, comprender, definir, apropiamos y valorar la salud y la enfermedad, la vida y la muerte, el riesgo y su prevención, implica explorar las relaciones que establecen entre sí y con la formación social hegemónica imperante donde el Estado juega un papel benefactor, o de subsidiario, o de regulador.

Durante la última década, México cursa por un proceso de transición en todos los ámbitos sociales como la salud, la educación, la seguridad social, propiciando una participación social que demanda satisfacción de necesidades socia- les, denuncia injusticia y desigualdad, o bien, propone esquemas alternativos de solución a través de planes, metas y enunciados doctrinarios. Estos elementos han propiciado que salud y economía, salud y desarrollo, establezcan una relación bidireccional que en la apertura económica mundial y de cara al siglo XXI, requiere de un estudio y análisis detenido en nuestro medio.

\section{Bibliografía}

Bustelo E.S., 1991: "La producción del estado de malestar. Ajuste y política social en América Latina” en Salud Pública N³3, México.

Bustamante-Montes L.P., Rascón-Pacheco RA, Borja-Aburto VH., 1990: "Efecto de la aplicación del indicador de años de vida productivos perdidos (modelo inversión producción consumo) en el ordenamiento de las causas de muerte en México 1990" en Rev Saúde Pública N² 28.

Drummond M., Stoddart G., Labelle M.A., Cushman R.., 1995: "Economía de la salud: introducción para médicos clínicos" En: Patricia Hernández (comp) Economía, Salud y Población (Economía de la Salud), México: Facultad de Economía, UNAM. 
Frenk J., Lozano R., González-Block M.A., et. al., 1994: Economia y salud: propuesta para el avance del sistema de salud en México. Informe final. México: Fundación Mexicana para la Salud.

Fundación Mexicana para la Salud, 1994: Bases doctrinarias de la reforma en salud, México: Fundación Mexicana para la Salud, 1994.

Gárate R., 1995: Ética y libertad, España: Universidad de Deusto. GomezDantes O. y Zorrilla P., 1994: La protección del consumidor en el campo de la salud, México: Fundación Mexicana para la Salud.

Hernández-Peña P., Zapata O., Leyva R. y Lozano T., 1991: “Equidad y salud: necesidades de investigación para la formulación de una política social” en Salud Pública $N^{\circ} 33$, México.

Lozano R., Murray C.J.L., Frenk J., Bobadilla J.L. y Fernández S., 1994: El peso de la enfermedad en México: un doble reto, México: Fundación Mexicana para la Salud.

Marin A., 1995: "Your money or your life?” En: Patricia Hernández (comp) Economia, salud y población (Economia de la Salud), México: Facultad de Economía, UNAM.

Molina Salazar R.E., Romero Velázquez J.R. y Trejo Rodríguez J.A., 1991: "Desarrollo económico y salud" en Salud Pública N 32, México.

Musgrove P., 1983: "La equidad del sistema de servicios de salud. Conceptos, indicadores e interpretación” en Bol Of Sanit $\mathrm{N}^{\circ}$ 95, Panam.

Musgrove P., 1993: "Reflexiones sobre las relaciones entre la salud y el desarrollo" en Bol Of Sanit $\mathrm{N}^{\circ} 114$, Panam.

Rice D.P., 1995: “Cooper BJ. El valor económico de la vida humana” En: Patricia Hernández (comp) Economía, salud y población (Economia de la Salud). México: Facultad de Economía, UNAM.

Rillo A.G., 1992: "Indicadores del proceso salud-enfermedad y las condiciones materiales de vida" en Revista de la Facultad de Medicina $\mathrm{N}^{\circ} 2$ : UAEM.

Rillo A.G., 1996: "Análisis teórico del informe final de FUNSALUD: "Economía y Salud" en La Mora N² 2, Toluca: UAEM.

Schalaepfer-Pedrazzini L. e Infante-Castañeda C., 1990: "La medición de salud: perspectivas teóricas y metodológicas” en Salud Pública $\mathrm{N}^{\circ} 32$, México.

Vandale Toney S., Durán Arenas L.G. y Ortega Minor H., 1985: "La medición del estado de salud de la población: una actividad fundamental para los servicios de salud" en Salud Pública N²7, México. 